\title{
MICROPHOTOMETRY-COMPUTER CLASSIFICATION OF OBJECTIVE-PRISM SPECTRA
}

\author{
K. M. YOSS \\ University of Illinois, Ill., U.S.A.
}

\begin{abstract}
Three-dimensional classification of late-type stars is obtained through computer-processed digitized microphotometer data. Plate transmissions are converted to relative intensity through spot-sensitometer calibration data. A pseudo-continuum consisting of several straight-line segments is formed by connecting high points in the spectrum. Absorption-line strengths and line ratios are then measured. The temperature-and-luminosity-sensitive ratios are relatively insensitive to seeing effects. For plates with a dispersion of $108 \AA \mathrm{mm}^{-1}$, preliminary results indicate an accuracy in derived absolute magnitude comparable to, and possibly better than, that of MK classification.

The $\mathrm{CN}$ anomaly serves as the abundance index. The $\mathrm{CN}$ index was defined in a manner to give the largest figure of merit (the ratio of total range of the index to the mean error). The adopted method is the same as that of Yoss and Lutz (1971) and has a figure of merit over twice that of the index similar to that of Griffin and Redman (1960).

For field stars, plate $\mathrm{X}$ and $\mathrm{Y}$ positions are converted to equatorial coordinates and printed out in order of increasing right ascension, making quick and easy identification of the Henry Draper numbers.
\end{abstract}

Three-dimensional classification of late-type stars is obtained through computer-processed digitized microphotometer data. Plates taken with the University of Michigan Curtis Schmidt, with a dispersion of $108 \AA \mathrm{mm}^{-1}$ at $\mathrm{H} \gamma$, and while the instrument was still in Michigan, were used for this preliminary study.

Plate transmissions are converted to relative intensity through spot-sensitometer calibration data. Feature identification depends on locating maximum or minimum points relative to a manually-recorded position of a particular identified feature. A pseudo-continuum consisting of several straight-line segments is formed by connecting high points in the spectrum (at 4027, 4091, 4221, 4245, 4318, and $4502 \AA$ ). Absorptionline strengths and line ratios are then measured. The temperature- and luminositysensitive ratios are relatively insensitive to seeing and sky-fog effects. Half a dozen ratios (for example, $4325 / 4340 \AA$ ) are used to derive spectral type to within one subclass.

The absolute-magnitude calibration depends on about 40 standard stars (generally two exposures per star) for which absolute magnitudes are known with mean errors $\leqslant \pm 0.5 \mathrm{mag}$. Three ratios $(4077 / 4071,4176 / 4132$, and $4215 / 4272 \AA)$ are found useful for deriving absolute magnitude, with mean errors of \pm 0.6 mag. (after the effects of the errors in the published values are removed). In spite of the fact that two of the ratios involve lines falling within the $\mathrm{CN}$ absorption band, there appears to be no dependence of the ratios on $\mathrm{CN}$ strength (or $\mathrm{CN}$ anomaly).

The $\mathrm{CN}$ anomaly serves as the abundance index. The $\mathrm{CN}$ index is defined in a manner to give the largest figure of merit (the ratio of total range of the index to the mean error of measurement). A two-segment CN continuum is fitted at 4091, 4163, and $4211 \AA$. 
The fractional absorption between the two continua is then the measured value, and the $\mathrm{CN}$ anomaly is the departure from the average value as defined by a linear line extending over the entire $M_{v}-\mathrm{CN}$ range. The figure of merit is 17 , compared to 8 for an index similar to that used by Griffin and Redman (1960) and 8 also for a visually estimated CN strength from similar plates (Yoss, 1961).

For aid in rapid identification of field stars, the plate $X$ and $Y$ positions are converted to equatorial coordinates and printed out in formats identical to the Henry Draper and BD catalogues. Generally prior identification of only one bright star in the field is sufficient to give coordinates accurate to $0.1 \mathrm{~min}$ in right ascension and to $2^{\prime}$ in declination. Derived spectral types and photographic magnitudes are also printed, to aid in identification. These magnitudes, determined from the summed relative energy curves of the spectra, with the zero point determined from the value for the identified star, generally agree to within $0.1 \mathrm{mag}$. of the Henry Draper values. Several stars can be identified per minute in this manner. The microphotometry takes less than two minutes per star.

\section{References}

Griffin, R. F. and Redman, R. D.: 1960, Monthly Notices Roy. Astron. Soc. 120, 287.

Yoss, K. M.: 1961, Astrophys. J. 134, 809.

Yoss, K. M. and Lutz, T. E.: 1971, Mem. Roy. Astron. Soc. 75, 21. 